\title{
TRAUMATIC SHOCK. VIII. STUDIES IN THE THERAPY AND HEMODYNAMICS OF TOURNIQUET SHOCK ${ }^{1}$
}

\author{
By JACOB FINE, HOWARD A. FRANK, AND ARNOLD M. SELIGMAN \\ (From the Surgical Research Department, Beth Israel Hospital, Boston, and \\ the Department of Surgery, Harvard Medical School, Boston)
}

(Received for publication November 11, 1943)

Although traumatic shock created by the release of a tourniquet applied for some hours to an extremity is an unusual clinical condition, it is comparable to other clinical states such as burns, intestinal obstruction, certain high velocity projectile wounds (1), etc., in which a large and more or less rapid loss of plasma from the circulation is the most obvious shock-inducing agent. While special features of these various conditions obscure the effects of the mere loss of plasma (e.g., in tourniquet and high velocity wounds, muscle is crushed; in burns, tissue is burned; and in simple intestinal obstruction, electrolytes and water are lost by vomiting), tourniquet shock constitutes a more controllable and possibly less complicated type than others for an experimental study of shock resulting primarily from plasma loss.

Numerous studies ( 2 to 7 ) of tourniquet shock are almost uniformly agreed that death usually occurs regardless of the therapy employed, unless the tourniqueted extremities are refrigerated $(5$, 6) immediately upon release of the tourniquets or tightly taped, so as to prevent the escape of plasma from the circulation (7). That taping is sufficient to prevent the onset of shock would argue against any relationship between the onset of shock and the muscle damage inflicted by the tourniquet at its site of application. It therefore is not surprising to note the failure to date of efforts to isolate an etiologically significant "toxin" from the crushed muscle. ${ }^{2}$ In these cir-

\footnotetext{
1 The work described in this paper was done under a contract, recommended by the Committee on Medical Research, between the Office of Scientific Research and Development and Harvard University.

2 The presence of $\mathrm{Cl}$. welchii in dog muscle has long been known. Aub et al. (A Toxic Factor in Experimental Traumatic Shock-New England J. Med., 1944, 231, 71) have frequently found this organism in large numbers in the fluid transudate from damaged muscle in the dog. Large quantities of such fluid were found to be toxic and
}

cumstances, it is all the more perplexing to find in the reports published to date almost uniform failure to achieve a cure of the shock state by early and adequate replacement of the plasma lost into the injured extremities.

A revaluation of the experimental set-up and the effectiveness of replacement therapy was therefore regarded as necessary. This report will present evidence that, if the proper circumstances are created, adequate replacement therapy is successful in an advanced state of shock. Data will also be given on the response to succinic acid therapy. Certain differences in response to blood volume replacement between hemorrhagic and tourniquet shock will be discussed.

\section{METHOD}

A review of the technics hitherto employed reveals that all experiments so far reported were carried out under anesthesia-usually a barbiturate. The notorious effect of anesthetics on the shock state led us to omit all anesthetics or sedatives except intravenous or intramuscular morphine sulphate ( 3 to $6 \mathrm{mgm}$. per $\mathrm{kgm}$.) which was given

capable of producing shock. Filtrates from cultures of $\mathrm{Cl}$. welchii isolated from muscle were also capable of inducing shock.

Such evidence does not establish a case for the etiological significance of this organism in tourniquet shock, for the following reasons: (1) Shock due to Welch toxin cannot be cured by plasma or albumin therapy. Tourniquet shock can be cured by these agents; (2) The death from tourniquet shock is extraordinarily rapid, a matter of a few hours usually, following release of the tourniquet, so that too little time is available for the development of enough toxin to be the lethal agent; (3) The amount of fluid transudate required by Aub et al. to induce fatal shock was usually far in excess of that produced by a single animal; (4) That Welch toxin is lethal is well known, but the type of shock produced, particularly the severe hemolytic effect of Welch toxin, is not seen in tourniquet shock; (5) Aub et al. found no correlation between the number of organisms cultured from damaged muscle and the severity of shock; some of these organisms in fact did not produce potent toxin; (6) Alteration of the course of tourniquet shock by pretreatment with antitoxin has not been demonstrated. 
TABLE I

Therapy of tourniquet shock

\begin{tabular}{|c|c|c|c|c|c|c|c|c|c|c|c|c|c|c|c|c|c|c|c|c|c|}
\hline \multirow{4}{*}{$\begin{array}{c}\begin{array}{c}\text { Experimental } \\
\text { groups }\end{array} \\
\\
\begin{array}{c}\text { A. } \\
\text { Physiol. saline } \\
\text { intravenoualy } \\
\text { (5 hour tourniquet } \\
\text { application) }\end{array}\end{array}$} & \multirow{4}{*}{$\begin{array}{c}\text { Group } \\
\text { result } \\
\\
\begin{array}{c}\text { Survived } \\
5 \text { of } 6\end{array}\end{array}$} & \multirow{4}{*}{$\begin{array}{l}\text { Suc- } \\
\text { cinio } \\
\text { acid } \\
\text { sur- } \\
\text { vival } \\
\\
\end{array}$} & \multirow{4}{*}{\begin{tabular}{|l|}
$\begin{array}{l}\text { Dog } \\
\text { no. }\end{array}$ \\
\\
\\
\\
S-68 \\
S-69 \\
S-70 \\
$8-71$ \\
S-72 \\
S-73 \\
\end{tabular}} & \multirow{4}{*}{$\begin{array}{c}\begin{array}{c}\text { Dog } \\
\text { wt. }\end{array} \\
\\
\text { kom. } \\
\\
10 \\
11.4 \\
15.5 \\
9.6 \\
19 \\
12.3\end{array}$} & \multirow{4}{*}{\begin{tabular}{|c}
$\begin{array}{c}\text { Tour- } \\
\text { niquet } \\
\text { on }\end{array}$ \\
hours \\
\\
5 \\
5 \\
5 \\
5 \\
5 \\
5
\end{tabular}} & \multicolumn{5}{|c|}{ Before treatment } & \multicolumn{11}{|c|}{ Therapy } \\
\hline & & & & & & \multirow{3}{*}{ Time* } & \multirow{3}{*}{$\mid \begin{array}{c}\mathrm{BP} \\
\mathrm{mm} . \\
\mathrm{B} . \\
\end{array}$} & \multirow{3}{*}{\begin{tabular}{|c|} 
Art. \\
hem.
\end{tabular}} & \multirow{3}{*}{\begin{tabular}{l|} 
P.V. \\
$m l$.
\end{tabular}} & \multirow{3}{*}{\begin{tabular}{c|} 
R.C.V.t \\
$m l$.
\end{tabular}} & \multirow{3}{*}{ 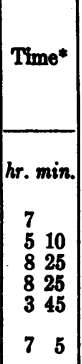 } & \multirow{3}{*}{\begin{tabular}{|c|}
$\mathrm{BP}$ \\
\\
$\mathrm{mm}$. \\
$\mathrm{Hg}$ \\
70 \\
75 \\
100 \\
115 \\
45 \\
65
\end{tabular}} & \multirow{3}{*}{$\begin{array}{l}\text { Art. } \\
\text { hem. } \\
\\
\\
74 \\
71 \\
48 \\
67 \\
70 \\
69\end{array}$} & \multirow{2}{*}{\multicolumn{2}{|c|}{$\begin{array}{c}25 \text { per } \\
\text { cent } \\
\text { bov. alb. }\end{array}$}} & \multicolumn{2}{|c|}{$\begin{array}{l}5 \text { per cent } \\
\text { bov. alb. }\end{array}$} & \multirow{2}{*}{\multicolumn{2}{|c|}{ Saline }} & \multicolumn{2}{|c|}{$\begin{array}{c}\text { Fluid } \\
\text { by stomach }\end{array}$} \\
\hline & & & & & & & & & & & & & & & & & & & & & Kind, \\
\hline & & & & & & & & & & & & & & $m l$. & $\begin{array}{c}\mathrm{ml} . \\
\text { per } \\
\mathrm{kgm} .\end{array}$ & $m l$. & $\begin{array}{c}\mathrm{ml} . \\
\text { per } \\
\mathrm{kgm} .\end{array}$ & $\begin{array}{r}m l . \\
1150 \\
2100 \\
750 \\
750 \\
3240 \\
1000\end{array}$ & 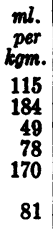 & $m l$. & \\
\hline $\begin{array}{l}\text { B. } \\
\text { Physiol. saline } \\
\text { intravenously } \\
\text { (10 hour tourniquet } \\
\text { application) }\end{array}$ & $\begin{array}{l}\text { Died } \\
6 \text { of } 6\end{array}$ & 0 of 6 & $\begin{array}{l}\text { S-51 } \\
\text { S-52 } \\
\text { S-53 } \\
\text { S-54 } \\
\text { 8-55 } \\
\text { S-56 }\end{array}$ & $\begin{array}{r}9.5 \\
10.0 \\
11.3 \\
14.0 \\
12.2 \\
10.9\end{array}$ & $\begin{array}{l}10 \\
10 \\
10 \\
10 \\
10 \\
10\end{array}$ & & & & & & \begin{tabular}{ll|}
2 & 10 \\
2 & 43 \\
2 & 50 \\
2 & 5 \\
2 & 45 \\
2 & 40
\end{tabular} & $\begin{array}{l}40 \\
40 \\
65 \\
40 \\
60 \\
60\end{array}$ & $\begin{array}{l}70 \\
58\end{array}$ & & & & & $\begin{array}{l}1100 \\
1300 \\
1550 \\
1600 \\
1350 \\
1600\end{array}$ & $\begin{array}{l}116 \\
130 \\
137 \\
114 \\
110 \\
147\end{array}$ & & \\
\hline $\begin{array}{c}\text { C. } \\
5 \text { per cent sodium } \\
\text { chloride intra- } \\
\text { venougly } \\
(20 \text { ml. per kgm ) } \\
+ \\
\text { water in stomach }\end{array}$ & $\begin{array}{l}\text { Died } \\
5 \text { of } 5\end{array}$ & 0 of 4 & $\begin{array}{l}\text { S-62 } \\
\text { S-63 } \\
\text { S-64 } \\
\text { S-65 } \\
\text { S-67 }\end{array}$ & $\begin{array}{l}5.5 \\
5.5 \\
8.0 \\
5.5 \\
5.9\end{array}$ & $\begin{array}{l}10 \\
10 \\
10 \\
10 \\
10\end{array}$ & & & & & & $\begin{array}{l}130 \\
2 \\
5 \\
130 \\
330\end{array}$ & $\begin{array}{l}60 \\
60 \\
70 \\
55 \\
60\end{array}$ & $\begin{array}{l}64 \\
67 \\
75 \\
81 \\
90\end{array}$ & & & & & $\begin{array}{l}110 \\
110 \\
160 \\
110 \\
120\end{array}$ & $\begin{array}{l}20^{\circ} \\
20 \\
20^{\circ} \\
20^{*} \\
20^{2}\end{array}$ & $\begin{array}{r}500 \\
1000 \\
1200 \\
650 \\
1200\end{array}$ & $\begin{array}{l}\text { Water } \\
\text { Water } \\
\text { Water } \\
\text { Water } \\
\text { Water }\end{array}$ \\
\hline $\begin{array}{l}\text { D. } \\
5 \text { per cent bovine } \\
\text { albumin } \\
(40 \text { to } 50 \mathrm{ml} \text {. per } \\
\text { kgm. })\end{array}$ & $\begin{array}{l}\text { Survived } \\
5 \text { of } 7\end{array}$ & 2 of 4 & $\begin{array}{l}\text { B-2 } \\
\text { B-3 } \\
\text { B-11 } \\
\text { B-12 } \\
\text { B-13 } \\
\text { B-14 } \\
\text { B-15 }\end{array}$ & $\begin{array}{r}12.7 \\
5.9 \\
10.0 \\
11.3 \\
18.2 \\
13.6 \\
8.2\end{array}$ & $\begin{array}{l}88 / 4 \\
83 / 4 \\
8 \\
8 \\
8 \\
8 \\
8\end{array}$ & 830 & 100 & 69 & 615 & 760 & $\begin{array}{cc}9 & 40 \\
4 & 20 \\
6 & 15 \\
13 \\
10 \\
6 \\
3 \\
3 & 35\end{array}$ & $\begin{array}{l}60 \\
40 \\
40 \\
60 \\
60 \\
70 \\
50\end{array}$ & $\begin{array}{l}73 \\
70 \\
70\end{array}$ & & & $\begin{array}{l}600 \\
250 \\
400 \\
500 \\
870 \\
565 \\
330\end{array}$ & $\begin{array}{l}47 \\
42 \\
40 \\
44 \\
48 \\
42 \\
40\end{array}$ & 200 & 34 & & \\
\hline $\begin{array}{l}\text { E. } \\
5 \text { per cent bovine } \\
\text { albumin } \\
\text { (unlimited) }\end{array}$ & $\begin{array}{l}\text { Survived } \\
6 \text { of } 7\end{array}$ & & $\begin{array}{l}\mathrm{B}-16 \\
\mathrm{~B}-17 \\
\mathrm{~B}-19 \\
\mathrm{~B}-21 \\
\mathrm{~B}-22 \\
\mathrm{~B}-24 \\
\mathrm{~B}-28\end{array}$ & $\begin{array}{l}14.5 \\
12.7 \\
13.2 \\
10.0 \\
10.9 \\
11.0 \\
10.0\end{array}$ & $\begin{array}{l}91 / 2 \\
11 / 2 \\
101 / 4 \\
10 \\
10 \\
9 \\
10\end{array}$ & $\begin{array}{ll}3 & 30 \\
6 & 30 \\
6 & 30 \\
5 & 15\end{array}$ & $\left|\begin{array}{r}90 \\
100 \\
60 \\
50\end{array}\right|$ & $\begin{array}{l}71 \\
67 \\
82 \\
71\end{array}$ & $\begin{array}{l}370 \\
440 \\
220 \\
180\end{array}$ & $\begin{array}{l}505 \\
550 \\
485 \\
280\end{array}$ & \begin{tabular}{rr|}
6 & 30 \\
4 & 11 \\
12 & 10 \\
6 & 42 \\
5 & 45 \\
5 & 10 \\
4 &
\end{tabular} & $\begin{array}{l}60 \\
30 \\
50 \\
60 \\
50 \\
60 \\
50\end{array}$ & $\begin{array}{l}63 \\
65\end{array}$ & & & $\begin{array}{r}800 \\
2275 \\
1000 \\
1200 \\
900 \\
1000 \\
1500\end{array}$ & $\begin{array}{r}55 \\
179 \\
76 \\
120 \\
83 \\
91 \\
150\end{array}$ & & & & \\
\hline
\end{tabular}

* Time from removal of tourniquets.

† Sodium chloride (5 grams per $\mathrm{ml}$.) added to albumin.

I Red cell volume determined with radioactive red cells by Gibson and Evans.

$\therefore$ Deviation of the plasma volume found after treatment, from the expected volume calculated on the basis of the intravenously administered fluid - expressed as a percentage of the plasma volume measured in shock.

35 per cent sodium chloride solution.

8 First noted time, blood pressure, and hematocrit, follow the 5 per cent saline therapy, and the second set of recordings refer to succinic acid therapy (section $\mathrm{C}$ ).

before and once or twice during the application of the tourniquets. The extremities became insensitive after some time and no further drug was necessary (a few exceptional instances required a single supplementary dose of $16 \mathrm{mgm}$. intramuscularly), so that the experiment thereafter was carried through with no further medication except therapeutic substances as noted below.

Following hairclipping, washing, and the application of 
TABLE I-Continued

Therapy of tourniquet shock

\begin{tabular}{|c|c|c|c|c|c|c|c|c|c|c|c|c|c|c|c|}
\hline \multicolumn{3}{|r|}{ Therapy } & \multicolumn{2}{|c|}{ Best response } & \multicolumn{9}{|c|}{ After treatment } & \multirow{3}{*}{ Result } & \multirow{3}{*}{$\begin{array}{l}\text { Pathological findings } \\
\text { and remarks }\end{array}$} \\
\hline & & Succinic acid & \multirow{2}{*}{ Time* } & \multirow{2}{*}{ BP } & \multirow{2}{*}{ Time* } & \multirow{2}{*}{ BP } & \multirow{2}{*}{$\begin{array}{l}\text { Art. } \\
\text { hem. }\end{array}$} & \multicolumn{3}{|c|}{ Placma volume } & \multicolumn{3}{|c|}{ Red cell volumeł } & & \\
\hline & line & When given & & & & & & Cala & Found & Net $=$ & Cale. & Found & Net & & \\
\hline $\begin{array}{c}\text { mom. } \\
\text { per } \\
\text { kgm. }\end{array}$ & ml. & & $\begin{array}{rrr}\text { hr. } & \text { min. } \\
11 & \\
6 & 5 \\
10 & 50 \\
11 & 15 \\
4 & 15 \\
7 & 30\end{array}$ & $\begin{array}{r}\operatorname{mon}_{g} \\
90 \\
95 \\
110 \\
120 \\
115 \\
105\end{array}$ & $\begin{array}{l}\text { hr. } \min . \\
1050 \\
1035 \\
11\end{array}$ & $\begin{array}{r}m m . \\
H_{0} \\
90 \\
80 \\
100\end{array}$ & $\begin{array}{l}52 \\
58 \\
48\end{array}$ & $m l$. & $m b$. & $\begin{array}{l}\text { por } \\
\text { cont }\end{array}$ & $m l$. & $m b$. & $m l$ & $\begin{array}{l}\text { Burvived } \\
\text { Survived } \\
\text { Survived } \\
\text { 8urvived } \\
\text { Died-22 hours } \\
\text { Survived }\end{array}$ & $\begin{array}{l}\text { Probably not in shock. } \\
\text { Probably not in shock. } \\
\text { Pulmonary congeation and ode- } \\
\text { ma. No hemorrhage in gut. }\end{array}$ \\
\hline $\begin{array}{r}89 \\
210\end{array}$ & & $\begin{array}{l}\text { Continuously } \\
\text { Continuously } \\
\text { Continuously } \\
\text { Continuously } \\
\text { Continuously } \\
\text { Continuously }\end{array}$ & $\begin{array}{l}3 \\
330 \\
445 \\
215 \\
445 \\
620\end{array}$ & $\begin{array}{r}90 \\
100 \\
90 \\
120 \\
110\end{array}$ & $\begin{array}{l}815 \\
930 \\
830 \\
515 \\
830 \\
825\end{array}$ & $\begin{array}{l}35 \\
40 \\
55\end{array}$ & $\begin{array}{l}52 \\
49 \\
47 \\
58 \\
62\end{array}$ & & & & & & & $\begin{array}{l}\text { Died-8 hours } 35 \text { minutes } \\
\text { Died-15 hours } \\
\text { Died-8 hours } 45 \text { minutes } \\
\text { Died-5 hours } 25 \text { minutes } \\
\text { Died-9 hours } \\
\text { Died-8 hours } 45 \text { minutes }\end{array}$ & 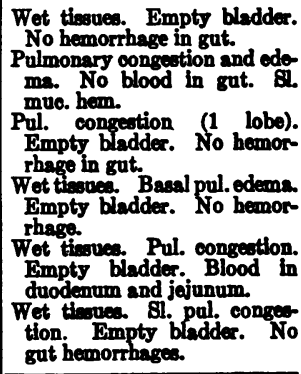 \\
\hline $\begin{array}{l}730 \\
850\end{array}$ & $\begin{array}{l}400 \\
500\end{array}$ & $\begin{array}{l}3 \text { hours at BP } 20 \\
4 \text { hours at BP } 30 \\
2 \text { hours } 40 \text { minutes } \\
\text { at BP } 20 \\
4 \text { hours } 50 \text { minutes } \\
\text { at BP } 35\end{array}$ & $\begin{array}{ll}1 & 405 \\
3 & 15 \\
2 & 30 \\
4 & 20 \\
5 & 25 \\
& \\
1 & 45 \\
3 & \\
3 & 40 \\
5 & 30\end{array}$ & $\begin{array}{r}90 \\
90 \\
75 \\
70 \\
85 \\
\\
75 \\
65 \\
110 \\
120\end{array}$ & $\begin{array}{ll}2 & 40 \\
4 & 40 \\
4 & 00 \\
5 & 25 \\
8 & 30 \\
& \\
2 & 40 \\
3 & 35 \\
4 & 50 \\
6\end{array}$ & $\begin{array}{l}20 \\
35 \\
30 \\
30 \\
75 \\
\\
20 \\
15 \\
35 \\
40\end{array}$ & $\begin{array}{l}53 \\
49 \\
66 \\
61 \\
69 \\
\\
75 \\
56 \\
81 \\
68\end{array}$ & & & & & & & $\begin{array}{l}\text { Died-5 hours } \\
\text { Died-6 hours } 10 \text { minutes } \\
\text { Died-24 hours } \\
\text { Died- } 4 \text { hours } 30 \text { minutes } \\
\text { Died-6 hours } 15 \text { minutes }\end{array}$ & $\begin{array}{l}\text { Pul. congeation and edema. } \\
\text { Fmpty bladder. Blood in } \\
\text { duodenum. } \\
\text { Pul. congeetion. Empty blad- } \\
\text { der. Hemorrhagic duodenum. } \\
\text { Stomach and gut empty. No } \\
\text { hemorr hage. } \\
\text { Empty bladder and gut. } \\
\text { Bloody bowal. Pul. conger- } \\
\text { tion. } \\
\text { Empty bladder. Large hemor- } \\
\text { rhage in large and small bowel. }\end{array}$ \\
\hline $\begin{array}{l}127 \\
40 \\
70\end{array}$ & & $\begin{array}{l}9 \text { hours } 15 \text { minutes } \\
\text { at BP } 50 \\
8 \text { hours } 15 \text { minutes } \\
\text { at BP } 80 \\
\text { with albumin } \\
\text { with albumin }\end{array}$ & $\begin{array}{rr}10 & 20 \\
5 & \\
6 & 35 \\
13 & 15 \\
11 & 30 \\
6 & 30 \\
7 & 35\end{array}$ & $\begin{array}{r}135 \\
80 \\
110 \\
110 \\
115 \\
105 \\
120\end{array}$ & $\begin{array}{ll}12 & 40 \\
11 & 30 \\
12 & \\
13 & 50 \\
11 & 30 \\
12 & 15 \\
12 & 30\end{array}$ & $\begin{array}{r}110 \\
70 \\
80 \\
95 \\
115 \\
80 \\
80\end{array}$ & $\begin{array}{l}54 \\
39 \\
46 \\
40 \\
51\end{array}$ & 1485 & 1180 & -48 & 760 & 820 & +60 & $\begin{array}{l}\text { Burvived } \\
\text { Survived } \\
\text { Died-20 hours } \\
\text { Died-28 hours } \\
\text { Burvived } \\
\text { Burvived } \\
\text { Burvived }\end{array}$ & $\begin{array}{l}\text { Pul. congestion and atelectads } \\
\text { Congeated liver. }\end{array}$ \\
\hline & & . & $\begin{array}{rr}6 & 45 \\
4 & 35 \\
14 & 15 \\
7 & 15 \\
5 & 55 \\
5 & 30 \\
5\end{array}$ & $\begin{array}{r}115 \\
90 \\
100 \\
120 \\
130 \\
95 \\
105\end{array}$ & 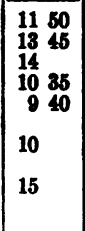 & $\begin{array}{r}110 \\
85 \\
100 \\
110 \\
95 \\
85 \\
105\end{array}$ & $\begin{array}{l}44 \\
80 \\
32 \\
83 \\
35 \\
44\end{array}$ & $\begin{array}{l}2645 \\
1440 \\
1490 \\
1080\end{array}$ & $\begin{array}{r}1030 \\
1130 \\
415 \\
410\end{array}$ & $\begin{array}{r}-436 \\
-70 \\
-456 \\
-372\end{array}$ & $\begin{array}{l}505 \\
550 \\
485 \\
760\end{array}$ & $\begin{array}{l}500 \\
540 \\
465 \\
345\end{array}$ & $\begin{array}{l}-5 \\
-10 \\
-20 \\
+85\end{array}$ & $\begin{array}{l}\text { Burvived } \\
\text { Died-16 hours } \\
\text { Burvived } \\
\text { Survived } \\
\text { Burvived } 82 \text { hours } \\
\text { Survived } \\
\text { Survived }\end{array}$ & $\begin{array}{l}\text { Cl. Welchil in leg museles. } \\
\text { Puruleqt infection of legs } \\
\text { (streptoococal). } \\
\text { Omentum exteriorised for mi- } \\
\text { oroecopy. } \\
\text { Omentum exteriorised for mi- } \\
\text { croscopy. }\end{array}$ \\
\hline
\end{tabular}

an antiseptic (tincture of iodine or zephiran), 5 or 6 turns of heavy-walled rubber tubing at maximal manual tension were applied to both legs as high on the thigh as possible and allowed to remain for 5 hours, the interval usually employed by previous investigators. Upon release, the extremities swelled, but shock was either slow to appear, i.e., frequently not for many hours (5 to 7), or it did not occur at all. Furthermore, the shock state so induced was effectively treated by saline solution alone. This was so contrary to all previous experience that the difference was regarded as due wholly to the omission of barbiturates. To induce rapid and severe shock, we altered the technic by merely prolonging the time of tourniquet application to 8 to 11 hours. Nearly all such dogs (79 out of 80 ) developed profound shock. If no treatment was given, death resulted usually in less than 6 hours and always in less than 12 hours.

Autopsy showed extensive muscle damage, with degeneration of muscle fibers and some hemorrhagic extravasation into the crushed muscle. The swelling distal to the 
TABLE I-Continued

\begin{tabular}{|c|c|c|c|c|c|c|c|c|c|c|c|c|c|c|c|c|c|c|c|c|}
\hline \multirow{4}{*}{ 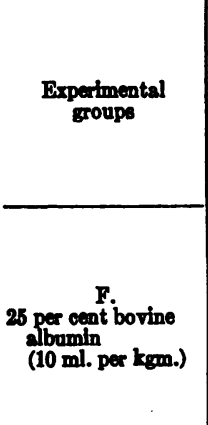 } & \multirow{4}{*}{$\begin{array}{l}\text { Group } \\
\text { result } \\
\\
\text { Died } \\
5 \text { of } 5\end{array}$} & \multirow{4}{*}{$\begin{array}{l}\text { Suc- } \\
\text { cinic } \\
\text { acid } \\
\text { sur- } \\
\text { vival } \\
0 \text { of } 3\end{array}$} & \multirow{4}{*}{$\begin{array}{l}\text { Dog } \\
\text { no. } \\
\\
\\
\text { B-5 } \\
\text { B-6 } \\
\text { B-7 } \\
\text { B-8 } \\
\text { B-9 }\end{array}$} & \multirow{4}{*}{$\begin{array}{c}\text { Dog } \\
\text { wt. } \\
\\
\text { kom. } \\
8.2 \\
7.8 \\
10 \\
11.4 \\
9.1\end{array}$} & \multirow{4}{*}{ 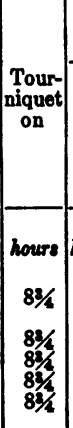 } & \multicolumn{5}{|c|}{ Before treatment } & \multicolumn{10}{|c|}{ Therappy } \\
\hline & & & & & & \multirow{3}{*}{\begin{tabular}{|c|} 
Time* \\
hr. min. \\
\end{tabular}} & \multirow{3}{*}{ BP } & \multirow{3}{*}{ Art. } & \multirow{3}{*}{\begin{tabular}{l|} 
P.V. \\
ml.
\end{tabular}} & \multirow{3}{*}{ R.C.V. } & \multirow{3}{*}{ 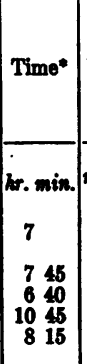 } & \multirow{3}{*}{\begin{tabular}{|c|} 
\\
\\
$B 9$ \\
40 \\
50 \\
50 \\
40 \\
60
\end{tabular}} & \multirow{3}{*}{ 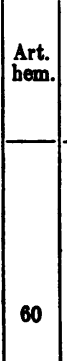 } & \multirow{2}{*}{\multicolumn{2}{|c|}{$\begin{array}{c}25 \text { per } \\
\text { cent } \\
\text { bov. alb. }\end{array}$}} & \multirow{2}{*}{\multicolumn{2}{|c|}{$\begin{array}{l}5 \text { per cent } \\
\text { bov. alb. }\end{array}$}} & \multirow{3}{*}{$\begin{array}{c}\text { Saline } \\
\text { ml. } \begin{array}{c}\text { ml. } \\
\text { per } \\
\text { kom. }\end{array}\end{array}$} & \multicolumn{2}{|c|}{$\begin{array}{c}\text { Fluid } \\
\text { by stomach }\end{array}$} \\
\hline & & & & & & & & & & & & & & & & & & & & Kind \\
\hline & & & & & & & & & & & & & & \begin{tabular}{|r|}
$m l$. \\
80 \\
80 \\
100 \\
110 \\
91
\end{tabular} & $\begin{array}{c}\text { ml. } \\
\text { per } \\
\text { kem. } \\
10 \\
10 \\
10 \\
10 \\
10\end{array}$ & $m l$. & $\left|\begin{array}{c}m l . \\
p \in r \\
k g m .\end{array}\right|$ & & $m l$. & \\
\hline $\begin{array}{l}\text { G. } \\
25 \text { per cent bovine } \\
\text { albumin } \\
\text { (10 ml. per kgm.) }+ \\
\text { saline in stomach }\end{array}$ & $\begin{array}{c}\text { Survived } \\
1 \text { of } 2\end{array}$ & & $\begin{array}{l}\text { B-25 } \\
\text { B-26 }\end{array}$ & $\begin{array}{l}11.8 \\
10.5\end{array}$ & $\begin{array}{l}111 / 2 \\
11 / 2\end{array}$ & 4 & 100 & 55 & 385 & 306 & $\begin{array}{l}5 \\
110\end{array}$ & $\begin{array}{l}60 \\
55\end{array}$ & & $\begin{array}{l}120 \\
105\end{array}$ & $\begin{array}{l}10 \\
10\end{array}$ & & & & $\begin{array}{l}1300 \\
1800\end{array}$ & $\begin{array}{l}\text { Saline } \\
\text { Saline }\end{array}$ \\
\hline $\begin{array}{c}\text { H. } \\
25 \text { per cent bovine } \\
\text { albumin } \\
(20 \mathrm{ml} \text {. per } \mathrm{kgm} \text {.) }\end{array}$ & $\begin{array}{l}\text { Died } \\
5 \text { of } 5\end{array}$ & & $\begin{array}{l}\mathrm{B}-38 \\
\mathrm{~B}-39 \\
\mathrm{~B}-40 \\
\mathrm{~B}-41 \\
\mathrm{~B}-42\end{array}$ & \begin{tabular}{r|}
12.3 \\
14.1 \\
11.4 \\
9.2 \\
11.8 \\
\end{tabular} & $\begin{array}{l}10 \\
10 \\
10 \\
10 \\
10 \\
\end{array}$ & 2 & $\begin{array}{l}90 \\
105\end{array}$ & $\begin{array}{l}77 \\
78\end{array}$ & $\begin{array}{l}240 \\
136\end{array}$ & $\begin{array}{l}480 \\
293\end{array}$ & $\begin{array}{ll}3 & 40 \\
2 & 20 \\
2 & 30 \\
3 & \\
3 & 10 \\
\end{array}$ & $\begin{array}{l}60 \\
65 \\
60 \\
55 \\
65\end{array}$ & $\begin{array}{l}77 \\
74 \\
80 \\
80 \\
77\end{array}$ & $\begin{array}{l}240 \\
280 \\
230 \\
180 \\
230\end{array}$ & $\begin{array}{l}20 \\
20 \\
20 \\
20 \\
20\end{array}$ & & & & & \\
\hline $\begin{array}{l}25 \text { per oent bovine } \\
\text { albumin } \\
(20 \mathrm{ml} \text {. per kgm.) } \\
\text { saline in stomsch } \\
\end{array}$ & $\begin{array}{c}\text { Survived } \\
5 \text { of } 6\end{array}$ & & $\begin{array}{l}\mathrm{B}-31 \\
\mathrm{~B}-32 \\
\mathrm{~B}-33 \\
\mathrm{~B}-34 \\
\mathrm{~B}-35 \\
\mathrm{~B}-36\end{array}$ & $\begin{array}{r}13.2 \\
11.4 \\
7.7 \\
9.2 \\
13.2 \\
10.0 \\
\end{array}$ & $\begin{array}{l}10 \\
10 \\
10 \\
10 \\
10 \\
10 \\
\end{array}$ & $\begin{array}{l}2 \\
220\end{array}$ & $\begin{array}{l}95 \\
95\end{array}$ & $\begin{array}{l}67 \\
66\end{array}$ & $\begin{array}{l}342 \\
220\end{array}$ & $\begin{array}{l}430 \\
221\end{array}$ & $\begin{array}{ll}4 & 30 \\
2 & 30 \\
3 & 30 \\
2 & 30 \\
5 & 10 \\
3 & 20\end{array}$ & $\begin{array}{l}60 \\
35 \\
70 \\
65 \\
65 \\
65 \\
\end{array}$ & $\begin{array}{l}60 \\
62 \\
69 \\
74\end{array}$ & $\begin{array}{l}264 \\
224 \\
153 \\
180 \\
264 \\
200\end{array}$ & $\begin{array}{l}20 \\
20 \\
20 \\
20 \\
20 \\
20 \\
\end{array}$ & & & & $\begin{array}{l}1250 \\
2600 \\
1250 \\
3300 \\
1250 \\
1250\end{array}$ & $\begin{array}{l}\text { Saline } \\
\text { Saline } \\
\text { Saline } \\
\text { Saline } \\
\text { Saline } \\
\text { Saline } \\
\end{array}$ \\
\hline $\begin{array}{l}\text { J. } \\
25 \text { per cent bovine } \\
\text { albumin } \\
(20 \mathrm{ml} \text {. per kgm.) } \\
+ \\
\text { water in stomach }\end{array}$ & $\begin{array}{l}\text { Survived } \\
\mathbf{3} \text { of } 6\end{array}$ & & $\begin{array}{l}\text { B-43 } \\
\text { B-44 } \\
\text { B-45 } \\
\text { B-46 } \\
\text { B-47 } \\
\text { B-48 }\end{array}$ & $\begin{array}{r}7.7 \\
6.8 \\
13.2 \\
9.2 \\
26.4 \\
18.2\end{array}$ & $\begin{array}{l}93 / 4 \\
93 / 4 \\
93 / 4 \\
93 / 4 \\
93 / 4 \\
93 / 4\end{array}$ & \begin{tabular}{ll|}
2 & 40 \\
2 & \\
3 & 13
\end{tabular} & $\begin{array}{r}100 \\
90 \\
90\end{array}$ & $\begin{array}{l}71 \\
62 \\
69\end{array}$ & $\begin{array}{l}290 \\
730 \\
455\end{array}$ & $\begin{array}{r}620 \\
1060 \\
702\end{array}$ & $\begin{array}{ll}2 & 10 \\
3 & 30 \\
6 & 10 \\
3 & 50 \\
7 & 30 \\
4 & 20\end{array}$ & $\begin{array}{l}55 \\
50 \\
60 \\
65 \\
75 \\
65\end{array}$ & $\begin{array}{l}67 \\
81 \\
\\
74 \\
67\end{array}$ & $\begin{array}{l}155 \dagger \\
136 \dagger \\
265 \dagger \\
180 \dagger \\
500 \dagger \\
364 \dagger\end{array}$ & $\begin{array}{l}20 \\
20 \\
20 \\
20 \\
20 \\
20\end{array}$ & & & & $\begin{array}{r}1500 \\
750 \\
500 \\
1950 \\
1650 \\
2000\end{array}$ & $\begin{array}{l}\text { Water } \\
\text { Water } \\
\text { Water } \\
\text { Watter } \\
\text { Water } \\
\text { Water }\end{array}$ \\
\hline $\begin{array}{l}\text { K. } \\
\text { Dog plasma (40 to } 50 \\
\text { ml. per kgm.) }\end{array}$ & $\begin{array}{c}\text { Survived } \\
2 \text { of } 2\end{array}$ & & $\begin{array}{l}4 \\
6\end{array}$ & $\begin{array}{l}14 \\
14.5\end{array}$ & $\begin{array}{r}91 / 4 \\
111 / 2\end{array}$ & & & & & & $\begin{array}{l}850 \\
7\end{array}$ & $\begin{array}{l}70 \\
70\end{array}$ & 70 & & & $\begin{array}{l}\text { (Plasma) } \\
\text { (Plasma) }\end{array}$ & 4 & & & \\
\hline
\end{tabular}

site of tourniquet application was pronounced, but it did not occur until after removal of the tourniquets. The arteries and veins were patent and there was no evidence of mechanical interference with blood flow during the shock state.

Following release of the tourniquets, no incision or other manipulation of the lower extremities was made, for it was evident early in our experience that such extremities were readily infected thereby. Accordingly, blood sampling and intravenous therapy were done under local anesthesia in the neck in most of the experiments reported below. The data gathered included determinations of arterial and venous (right heart) oxygen concentration, oxygen consumption (tracheal cannula inserted under local anesthesia), cardiac output (8), arterial hematocrit and arterial blood pressure (carotid artery), before shock and before and after therapy during shock. In some experiments, plasma and red cell volumes were measured, ${ }^{3}$ using the dye technic and red cells tagged with radioactive iron.

The experiments were usually carried out by studying

These data, gathered, in collaboration with Gibson and Evans, for the purpose of determining peripheral vascular stagnation, will appear elsewhere. a given type of therapy in 5 or 6 dogs simultaneously, thus eliminating variations in response to tourniquet application, which seem to depend on environmental conditions. (Thus we have the impression that dogs go into very rapid and severe shock more readily on hot humid days.)

After release of the tourniquets, the dogs became quiet and apathetic, the legs swelled rapidly, and shock was obviously present before the blood pressure began to fall, as indicated by the dull apathetic state of the animal and an already markedly lowered cardiac output (8). In the 61 dogs of this report, therapy was started only when the blood pressure had fallen to $70 \mathrm{~mm}$. $\mathrm{Hg}$ or lower. While this level of blood pressure is regarded as "the critical level" in hemorrhagic shock, a higher level is critical for tourniquet shock. Hence, the treatment may be regarded as having been applied after poor blood flow had been present for some time.

The therapy given was intended only for cure of the shock state. Successful therapy is herewith defined as recovery from shock and survival for a minimum of 24 hours after removal of the tourniquets, regardless of whether or not the dog subsequently died or was sacrificed because of massive necrosis or sepsis. It is possible that 
THERAPY AND HEMODYNAMICS OF TOURNIQUET SHOCK

TABLE I-Continued

\begin{tabular}{|c|c|c|c|c|c|c|c|c|c|c|c|c|c|c|c|}
\hline \multicolumn{3}{|r|}{ Therapy } & \multicolumn{2}{|c|}{ Best response } & \multicolumn{9}{|c|}{ After treatment } & \multirow{3}{*}{ Reoult } & \multirow{3}{*}{$\begin{array}{l}\text { Pathological findings } \\
\text { and remarks }\end{array}$} \\
\hline & & Succinic acid & \multirow{2}{*}{ Time* } & \multirow{2}{*}{ BP } & \multirow{2}{*}{ Time* } & \multirow{2}{*}{ BP } & \multirow{2}{*}{$\begin{array}{l}\text { Art. } \\
\text { hem. }\end{array}$} & \multicolumn{3}{|c|}{ Plasma volume } & \multicolumn{3}{|c|}{ Red cell volumet } & & \\
\hline & line & When given & & & & & & Calc. & Found & Nets & Calc. & Found & Not & & \\
\hline \multirow[t]{5}{*}{$\begin{array}{c}\text { mom } \\
\text { kgm } \\
100 \\
100 \\
80\end{array}$} & $\begin{array}{l}m l . \\
200 \\
200 \\
100\end{array}$ & $\begin{array}{l}\text { after albumin BP } 40 \\
\text { after albumin BP } 40 \\
\text { after albumin BP } 40\end{array}$ & \begin{tabular}{rr|}
7 & 15 \\
8 & 40 \\
7 & 10 \\
14 & 15
\end{tabular} & $\begin{array}{r}m g . \\
B g \\
95 \\
100 \\
90 \\
90 \\
90\end{array}$ & hr. min. & $\min _{g}$ & & $\mathrm{ml}$. & $m l$. & $\begin{array}{l}\text { per } \\
\text { cent }\end{array}$ & $m b$. & $m b$. & $m l$. & $\begin{array}{l}\text { Died-13 hours } 20 \text { minutes } \\
\text { Died-15 hours } 45 \text { minutes } \\
\text { Died-13 hours } 45 \text { minutes } \\
\text { Died-28 hours } \\
\text { Died-24 hours }\end{array}$ & $\begin{array}{l}\text { Small amount of blood in } \\
\text { jejunum. } \\
\text { No blood in gut. } \\
\text { No blood in gut. } \\
\text { (One tourniquet off too early.) } \\
\text { Omentum exteriorised. }\end{array}$ \\
\hline & & & $\begin{array}{l}540 \\
440\end{array}$ & $\begin{array}{r}100 \\
85\end{array}$ & $\mathbf{1 2}^{30}$ & $\begin{array}{l}95 \\
65\end{array}$ & $\begin{array}{l}34 \\
43\end{array}$ & 505 & 250 & +115 & 306 & 332 & +26 & $\begin{array}{l}\text { Survived } \\
\text { Died-28 hours }\end{array}$ & $\begin{array}{l}\text { Loft bronohopneumonis. Cl. } \\
\text { Welchil in legs. }\end{array}$ \\
\hline & & & $\begin{array}{lr}4 & 25 \\
2 & 40 \\
2 & 40 \\
3 & 5 \\
5 & 10\end{array}$ & $\begin{array}{l}125 \\
130 \\
100 \\
110 \\
105\end{array}$ & $\begin{array}{ll}6 & \\
6 & 35 \\
7 & 5 \\
6 & \\
8 & \end{array}$ & $\begin{array}{l}95 \\
35 \\
55 \\
55 \\
85\end{array}$ & $\begin{array}{l}55 \\
68 \\
68 \\
54 \\
62\end{array}$ & $\begin{array}{l}480 \\
316\end{array}$ & $\begin{array}{l}460 \\
220\end{array}$ & $\begin{array}{l}-8 \\
-71\end{array}$ & $\begin{array}{l}480 \\
298\end{array}$ & $\begin{array}{l}480 \\
203\end{array}$ & $\begin{array}{r}0 \\
-60\end{array}$ & $\begin{array}{l}\text { Died-15 hours } \\
\text { Died-6 hours } 45 \text { minutes } \\
\text { Died-7 hours } 35 \text { minutes } \\
\text { Died-6 hours } 40 \text { minutes } \\
\text { Died-13 hours }\end{array}$ & Accidental blood loss corrected. \\
\hline & & & $\begin{array}{ll}5 & 30 \\
6 & \\
3 & 45 \\
3 & \\
6 & 20 \\
7 & 40\end{array}$ & $\begin{array}{r}105 \\
80 \\
110 \\
85 \\
100 \\
130\end{array}$ & \begin{tabular}{rr|}
7 & 30 \\
11 & \\
6 & 20 \\
9 & \\
8 & 30 \\
7 & 10
\end{tabular} & $\begin{array}{r}100 \\
55 \\
100 \\
85 \\
100 \\
130\end{array}$ & $\begin{array}{l}31 \\
48 \\
34 \\
39 \\
37 \\
46\end{array}$ & $\begin{array}{l}606 \\
373\end{array}$ & $\begin{array}{l}805 \\
540\end{array}$ & $\begin{array}{l}+58 \\
+76\end{array}$ & $\begin{array}{l}430 \\
221\end{array}$ & $\begin{array}{l}420 \\
212\end{array}$ & $\begin{array}{l}-10 \\
-9\end{array}$ & $\begin{array}{l}\text { Survived } \\
\text { Diod-13 hours } \\
\text { Survived } \\
\text { Survived } \\
\text { Survived } \\
\text { Survived }\end{array}$ & \\
\hline & & & $\begin{array}{cc}2 & 20 \\
1 & 20 \\
7 \\
4 \\
10 \\
5\end{array}$ & $\begin{array}{l}105 \\
140 \\
105 \\
110 \\
100 \\
110\end{array}$ & \begin{tabular}{rr|}
8 & 40 \\
8 & 15 \\
7 & 50 \\
8 & 30 \\
10 & \\
7 & 35
\end{tabular} & $\begin{array}{r}100 \\
35 \\
95 \\
120 \\
100 \\
100\end{array}$ & $\begin{array}{l}45 \\
68 \\
52 \\
48 \\
40 \\
40\end{array}$ & $\begin{array}{r}555 \\
1250 \\
819\end{array}$ & $\begin{array}{r}621 \\
1820 \\
730\end{array}$ & $\begin{array}{l}+23 \\
+81 \\
-20\end{array}$ & $\begin{array}{r}620 \\
1060 \\
702\end{array}$ & $\begin{array}{r}620 \\
1105 \\
478\end{array}$ & $\begin{array}{r}0 \\
+45 \\
-224\end{array}$ & $\begin{array}{l}\text { Died-24 hours } \\
\text { Died-8 hours } 20 \text { minutes } \\
\text { Survived } \\
\text { Survived } \\
\text { Survived } \\
\text { Died-11 hours }\end{array}$ & $\begin{array}{l}\text { No water or blood in gut. } \\
\text { Empty bladder and inteatines } \\
\text { Hemorrhage in duodenum. } \\
\text { Empty bladder. Markedly dig- } \\
\text { tended gut ( } 2000 \mathrm{ml} .+) \text {. No } \\
\text { blood in gut. }\end{array}$ \\
\hline 57 & & $\begin{array}{l}2 \text { hours and } 4 \text { hours } \\
\text { after plasma }\end{array}$ & $\begin{array}{l}9 \\
720\end{array}$ & $\begin{array}{l}150 \\
110\end{array}$ & $\begin{array}{l}1220 \\
13\end{array}$ & $\begin{array}{c}110 \\
105\end{array}$ & 49 & & & & & & & $\begin{array}{l}\text { Survived } \\
\text { Survived }\end{array}$ & \\
\hline
\end{tabular}

* Time from removal of tourniquets.

† Sodium chloride ( 5 grams per $100 \mathrm{ml}$.) added to albumin.

¥ Red cell volume determined with radioactive red cells by Gibson and Evans.

Deviation of the plasma volume found after treatment, from the expected volume calculated on the basis of the intravenously administered fluid-expressed as a percentage of the plasma volume measured in shock.

- 5 per cent sodium chloride solution.

8 First noted time, blood pressure, and hematocrit follow the 5 per cent saline therapy, and the second set of recordings refer to succinic acid therapy (section C).

an occasional dog which died before the 24-hour period succumbed in part to sepsis, since streptococci and $\mathrm{Cl}$. welchii were found in the leg muscles of many and in the heart's blood of some of these dogs. Such dogs are listed as dead from shock. At the termination of each experiment, sulfanilamide powder was placed in all wounds before closure.

The therapy given was of 5 types: (1) Crystallized bovine serum albumin," given intravenously in 25 per cent

- The crystallized bovine serum albumin employed in this work was prepared at the Armour Laboratories, Chicago, Illinois, by the method of Cohn and Hughes, under a contract, recommended by the Committee on Medical Research, of the Office of Scientific Research and Development.

Concentrated human serum albumin was recommended to the armed forces by the Subcommittee on Blood Substitutes of the National Research Council in the solution containing 1.5 per cent $\mathrm{NaCl}$, or in 5 per cent solution containing 0.9 per cent $\mathrm{NaCl}$. (2) In certain experiments with 25 per cent bovine albumin, water or

interests of convenience for transport and injection. The directions for its use state:

"Marked dehydration-As albumin draws fluid into the blood stream at the expense of other body fluids, patients who are severely dehydrated need additional water and salt. This may be given orally, if tolerated, intravenously, or by any other available route."

On each albumin bottle is stamped:

"Precaution-In the presence of dehydration, albumin must be given with or followed by additional fluids."

(See Woodruff, L. M., and Gibson, S. T., U. S. Naval Med. Bull., 1942, 40, 791, and Newhouser, L. R., and Lozner, E. L., Ibid., 796.) Insofar as the experimental results herewith reported can be applied to shock in man, the above precaution seems eminently justified. 
0.9 per cent $\mathrm{NaCl}$. was given by stomach tube and repeated to the limit of tolerance, i.e., gastric capacity. Where vomiting occurred, the net volume retained is listed as the amount given (Table I). (3) Saline solution, 0.9 per cent, intravenously. (4) Saline solution, 5 per cent, intravenously, together with water by stomach tube. (5) Succinic acid (C.P.) in the doses specified below, dissolved in water by neutralization with $\mathrm{NaOH}$. It was given with or following intravenous bovine albumin or saline solution. A volume of saline solution sufficient to raise the blood pressure above $80 \mathrm{~mm}$. $\mathrm{Hg}$ was given when required to ensure as far as possible adequate peripheral vascular distribution of the succinic acid.

\section{Group $A$}

RESUlts (see Table I)

As already stated, the almost uniformly fatal shock which most previous investigators report as the result of the application of tourniquets to both legs for a period of 5 hours regardless of the kind of therapy contrasts with our observation that shock is either mild or does not occur providing that no barbiturate or other form of anesthesia except initial doses of morphia are used. In Table I, Group A, 6 dogs, so prepared, are shown to have maintained their blood pressure at generally higher levels and for a longer period following removal of the tourniquets, before any treatment was given, than all other groups listed. Moreover, 5 of the 6 dogs recovered, all 6 having received 0.9 per cent $\mathrm{NaCl}$ solution intravenously in varying doses. Only Dog S-72, which died after 22 hours with pulmonary edema, was in deep shock when treatment was started. Two dogs, S-70 and S-71, were apparently not in shock. The other 3 were in mild shock and the response in all but one to saline therapy was quick and adequate in respect to blood pressure and fall of hematocrit. Edema of the legs was considerable in all 6 dogs.

\section{Comment}

This group, plus a number of similarly prepared dogs previously observed, convinced us that while 5-hour tourniquet shock plus barbiturate anesthesia is a relatively irreversible type of shock, 5-hour tourniquet shock without anesthesia is mild and yields readily to saline infusion only.

The successful treatment of tourniquet shock in nembutalized dogs by the use of enormous doses of intravenous saline solution has been reported $(9,10)$. One view is that a fatal depletion of blood volume results from the increased fluid demands of the damaged tissues (inflammation and increased capillary filtration). If these demands are met by an admittedly nearly fatal volume of saline solution, the depletion of the circulation will stop and recovery from shock will always or nearly always result. Others explain the recovery as due to an increase in pressure of the markedly edematous tissues throughout the body, sufficient to halt the further escape of intravenously injected salt solution (and protein) from the circulation. It is possible however that the recovery may be attributable to the dilution of barbiturate or to the substantial urinary excretion of the barbiturate and the consequent conversion of a relatively irreversible to a readily reversible type of shock.

\section{Group B}

The dogs of Group B were prepared like those of Group A except that the tourniquets remained on for 10 hours. They received 0.9 per cent saline solution intravenously in about the same doses as those of Group A and, in addition, succinic acid in an initial dose and by subsequent continuous drip, sufficient to maintain a minimum intravascular concentration of $44 \mathrm{mgm}$. per cent. $^{5}$ The shock was profound in all 6 in less than 3 hours following tourniquet release. All 6 died, 5 in 9 hours or less. In spite of the large volume of saline given, the bladders were empty, showing absence of renal function. Noteworthy is the observation that the tissues were wet in 4 , in 4 , the lungs were "congested", and in 2 , there was hemorrhagic extravasation into the gut.

\section{Group $C$}

The 5 dogs of this group, whose tourniquets were released after 10 hours, received $20 \mathrm{ml}$. per $\mathrm{kgm}$. of 5 per cent $\mathrm{NaCl}$ intravenously to provide an osmotic tension equivalent to that of $20 \mathrm{ml}$. per $\mathrm{kgm}$. of 25 per cent bovine albumin used in other groups of experiments. Tap water (500

\footnotetext{
- This amount is recommended (11) to provide satisfactory conditions, in a partially or completely anaerobic environment, for maximum utilization of an energy providing substrate which can be burned while glucose cannot be.
} 
to $1200 \mathrm{ml}$.) by gastric tube was added. The improvement in the shock state was slight and transitory, so that, within 1 to 3 hours, the blood pressures were below $35 \mathrm{~mm}$. Hg. Succinic acid was then given (750 to $850 \mathrm{mgm}$. per $\mathrm{kgm}$. in 400 to $500 \mathrm{ml}$. saline) in a large initial dose, followed by continuous drip as in Group B. Improvement was likewise transitory and 4 dogs were dead within 6.2 hours and 1 in 24 hours. Four of the 5 dogs had hemorrhagic bowel, 3 had pulmonary congestion, and 4 had empty bladders.

\section{Comment}

We have repeatedly noted the fact that dogs in hemorrhagic or tourniquet shock treated unsuccessfully with intravenous solutions show wet tissues or hemorrhagic intestinal mucosa or both, whereas dogs dying of shock (burn shock excepted) which have received no intravenous fluids do not show wet tissues or hemorrhagic intestinal mucosa. It is our inference that wet tissues are due to a failing capillary circulation which cannot resorb, via the venous end of the capillary, a solution which readily escapes from the arterial end of the capillary and that lymphatic return of tissue fluids to the circulation (except perhaps in burns) likewise fails. It is not being argued that wet tissues are due to greater permeability of capillaries to water; rather that the balance between the normal rate of escape and return of water may be upset. The question of disturbed capillary permeability resulting in leakage of plasma was answered in the negative on grounds previously offered (12 to 14), and by the additional evidence herewith provided that, while wet tissues are readily produced by saline infusions, they are less readily produced by infusions of concentrated albumin, whole blood, or plasma. The evidence is that wet tissues are not a phenomenon of shock, but are the result of the fluid therapy of shock and that saline solution is far more prone to produce the effect than whole blood or plasma.

\section{Group D}

Seven dogs (tourniquets for 8 hours), when in deep shock, were given 40 to $50 \mathrm{ml}$. per $\mathrm{kgm}$. of 5 per cent bovine albumin solution in a single transfusion lasting $1 \frac{1}{2}$ hours. The dose was equal to two-thirds of the estimated original total plasma volume and may therefore be regarded as adequate, not only to replace loss, but to provide an additional reserve for a continuing loss into the lower extremities. Four received succinic acid, 2 with the albumin and 2 afterwards. Five of the 7 dogs survived. Of these 5,2 received succinic acid; 3 did not. Both dogs which died also received succinic acid but possibly not in adequate dosage. Their survival was 20 and 28 hours, respectively.

\section{Comment}

The evidence is clear that the albumin was therapeutically effective and that the succinic acid probably was not.

\section{Group $E$}

Of 7 dogs which received 5 per cent bovine albumin, as in Group D, but in which supplementary sustaining doses were added as circumstances required, 6 recovered from shock. The seventh was treated only after the blood pressure had fallen precipitously to $30 \mathrm{~mm}$. $\mathrm{Hg}$., i.e., in a preterminal phase. None of these dogs received succinic acid.

\section{Group $F$}

All 5 dogs given 25 per cent bovine albumin in a dose of $10 \mathrm{ml}$. per $\mathrm{kgm}$. (equivalent to $50 \mathrm{ml}$. per $\mathrm{kgm}$. of the 5 per cent solution) died. Three of these received succinic acid in saline solution 3 to 4 hours after the albumin had been given and had failed to improve the shock state.

\section{Group $G$}

Two more dogs were treated as in Group F. No succinic acid was given, but saline solution was fed by stomach tube. One died and one recovered.

\section{Group $H$}

All 5 dogs, treated with a dose of $20 \mathrm{ml}$. per $\mathrm{kgm}$. of 25 per cent albumin and no succinic acid, died.

\section{Group I}

Of 6 dogs treated as in Group $\mathrm{H}$ but with saline solution by stomach tube, 5 survived. 
The sixth was first treated when the blood pressure had dropped precipitously to $35 \mathrm{~mm}$. $\mathrm{Hg}$.

\section{Group J}

Of 6 dogs treated as in Group I except that water instead of saline solution was given by mouth, 3 survived. The absorption of water was inadequate in dog B-48. Of the $3 \mathrm{dogs}$ which died, 2 had the lowest blood pressures in the group (50 and 55). These experiments were run on an unusually hot and humid day.

\section{Comment}

Whereas the dogs in Group $\mathrm{H}$ (25 per cent bovine albumin alone) partly restored the hematocrit toward normal, those in Group I (25 per cent bovine albumin plus peroral saline solution) showed a drop to even lower than normal levels, presumably because saline solution, made available by stomach tube, was effectively absorbed and retained within the circulation. The result was similar to that of Group E (5 per cent bovine albumin), in which the saline was given in almost comparable amounts with the albumin, intravenously.

It appears (1) that merely introducing osmotically effective proteins into the blood stream in the absence of a reservoir of fluids upon which to draw for the maintenance of blood volume will not constitute successful therapy; (2) that either the tissues lack an adequate fluid reservoir before shock is induced or that this reservoir, however adequate, is pre-empted by the fluid demands of the injured extremities.

The practical effect of these observations, so far as utilization of concentrated plasma and blood substitutes for shock in the field is concerned, is obvious. Supplementary parenteral or peroral fluids must be supplied. Whether the fluid given by mouth should be saline solution or tap water is not settled. In this connection, it should be clear that absorption of fluids from the intestine in shock is deficient (15).

\section{Group $K$}

Two dogs which were given plasma in a dose of $40 \mathrm{ml}$. per $\mathrm{kgm}$. survived.

\section{DISCUSSION OF RESULTS}

It is thus evident that unanesthetized dogs subjected to tourniquet trauma to both extremities for 5 hours will survive if given merely saline solution intravenously ( 5 survivals in 6 experiments). On the other hand, if the trauma is applied for 8 or 11 hours, all dogs will die whether untreated, treated by intravenous physiological saline solution, or 5 per cent saline solution supplemented with water by stomach tube. The only difference observed in 8 to 11-hour tourniquet shock between the saline treated animals and those not treated was the presence in the former and not in the latter of wet or hemorrhagic tissues (especially the lungs and intestine). It was clear that the saline solution failed to restore renal function, since no urine was excreted during the shock phase in spite of the administration of well over a liter of fluids in each case. The saline solution obviously left the capillaries. Its failure to return in substantial degree to the circulation is evidence of a deficient venous capillary and lymphatic flow. Since hemorrhage in the gut and other tissues was not observed in untreated tourniquet shock, the escape of red cells must also be attributed to intravenous fluid therapy, especially to saline solution. Therefore, when an advanced state of tourniquet shock in the absence of anesthesia exists, the use of intravenous saline solution alone must be regarded as having disadvantages without sufficient compensating advantages.

On the other hand, when an osmotically active protein, such as 25 per cent bovine albumin, is given, saline intravenously or orally is in part retained within the circulation. In these circumstances, hemorrhages into the intestine and wet tissues are only rarely seen.

The importance of saline solution as a supplement to protein therapy is shown by the fact that 10 dogs receiving 25 per cent bovine albumin without supplementary saline all died; whereas 5 of 6 dogs receiving supplementary saline solution by stomach tube survived, and 11 of 14 dogs receiving 5 per cent bovine albumin in saline solution also survived.

The characteristically high hematocrit values of tourniquet shock were almost uniformly reduced to normal or less than normal values when therapy was successful. This was as a rule not 
the case when therapy failed, whether the therapy was intravenous saline solution alone, 25 per cent albumin solution alone, or 25 per cent albumin and fluid by stomach tube. In the case of intravenous saline solution alone, the insufficiently reduced hematocrit was due to failure of intravascular retention of the infused fluid. In the case of 25 per cent albumin solution alone, it was due to the absence of an available interstitial fluid reservoir, for the 25 per cent albumin solution exerted a satisfactory osmotic effect, as is evident from the extraordinarily dry state of the tissues. When 25 per cent albumin intravenously was supplemented by saline solution via a stomach tube, the saline was effectively absorbed and retained by the circulation, as indicated by the extent of the rise in plasma volume. Thus, in Group $\mathrm{H}$ (25 per cent albumin solution alone), dogs B-38 and 41 failed to improve plasma volume above that of the fluid given, whereas in Group J (25 per cent albumin plus saline solution by stomach tube), dogs B-31 and B-33 showed a plasma volume in excess of the expected amount by 25 per cent and 34 per cent, respectively.

A fall in hematocrit to a normal or less than normal level accompanied the increase in plasma volume in 10 instances in which such data were obtained. (The hematocrit change cannot be - used to calculate the change in plasma volume.) Eight of these 10 dogs survived.

From the foregoing data, it appears that blood substitutes in the form of plasma, 5 per cent bovine albumin, or 25 per cent bovine albumin plus supplementary fluids by stomach tube, are effective modes of therapy in tourniquet shock. There were 30 dogs which received such therapy.
Seventy-three per cent (22 of 30 ) recovered. In 6 of the 8 which died, therapy was applied at a blood pressure below 60 . Only 5 of the 22 which survived received therapy after the blood pressure had dropped below 60. Dogs in tourniquet shock are in an advanced state of collapse when the blood pressure is 70 . A mean pressure of 60 may therefore be regarded as close to the permanently irreversible level in tourniquet shock.

The conclusion from the fact that effective replacement of blood volume is curative in tourniquet shock is that this type of shock results from oligemia and is not primarily toxigenic. To what extent toxins liberated from damaged muscle or ischemic tissues play an adjuvant rôle cannot be assessed. That they are of minor consequence is clear (1) from the fact that absorption of toxins, if present, is not interfered with, since circulation through the damaged area proceeds satisfactorily, and (2) because taping prevents shock by preventing loss of blood volume into the leg, without interfering with absorption. There is, accordingly, no clearly established difference between hemorrhagic and tourniquet shock in respect to the agents responsible for initiating the shock state. Necrosis and infection which occur following recovery from tourniquet shock are sequel or adjuvant phenomena bearing no relationship to the rapid collapse immediately pursuant to the release of the tourniquets.

Significant differences between the phenomena of hemorrhagic and tourniquet shock do exist however and these modify the course of events as well as the kind of therapy that is applicable in each. These differences may be tabulated as follows:
HEMORRHAGIC SHOCK

Fluid loss into localized areas Blood deficiency

Hematocrit

Critical level of mean blood pressure

Relationship of level of blood pressure and its duration to curability Capillary circulation

Sensorium

Tolerance to blood sampling
None

Whole blood

Normal or low

50 to 70 or less and comparatively well tolerated for hours before irreversible collapse occurs

\pm 20 for hours

Slow flow and capillaries contain few red cells

Dull at blood pressures much below 70

Good until very low pressures are reached
TOURNIQUET SHOCK

Large

Plasma-slight volume of red cells 65 to 85 per cent

100 or less-levels below 70 poorly tolerated and followed shortly by rapid and irreversible collapse

Below $\mathbf{4 0}$ for minutes

Slow flow, but capillaries contain many red cells

Dull at pressures below 100

Poor at pressures between 80 and 100 
The most remarkable physiological differences are the level of blood pressure at which shock may be said to exist (the critical blood pressure), the tolerance to bleeding at low pressures, and the hematocrit level. It is pertinent here to emphasize these differences from the point of view of (1) the kind of therapy necessary and (2) the time in shock when therapy may still be effective.

Since whole blood will only slightly decrease the hematocrit in tourniquet shock, its use, while perhaps not damaging, would not facilitate as quick a response as plasma or albumin plus saline solution, because the continuing high hematocrit would continue to maintain too high a viscosity for maximum cardiac output.

As already indicated, the blood pressure level is grossly misleading in tourniquet shock. Upon release of the tourniquets, the legs rapidly swell and even though a large loss of plasma has already occurred, the blood pressure tends to remain at some $100 \mathrm{~mm}$. $\mathrm{Hg}$ for an hour or two. But the dog is obviously sick. The cardiac output in experiments to be reported (8) was found to be as low as 25 per cent of normal and the venous oxygen 5 volumes per cent within 15 minutes of release of the tourniquets. Once the blood pressure begins to fall, it falls precipitously. To withhold therapy until levels considered reasonably safe in hemorrhagic shock are reached is likely to be disastrous. Therapy at pressures of 100 to 70 however may be expected to be effective.

The relationship between high hematocrit and viscosity is of fundamental significance in the therapy of burn shock, dehydration shock, and other clinical states in which plasma is lost out of proportion to red cells. Data on the extent of the parallelism between viscosity and hematocrit have been gathered (16), as measured by slow and high velocity viscometers and by perfusion of the dog's hind limb. Data by the high velocity viscometer method approximate those of hind limb perfusion. Using the high velocity viscometer method, we have repeated this study on blood from tourniquet shock dogs and correlated the results with the cardiac output. Within the range of clinically encountered hematocrit values, the correlation is one of a progressive fall in cardiac output with a rise in hematocrit (viscosity) providing blood pressure and blood volume are kept constant. The full discussion of this problem will appear in another publication (8). The foregoing may suffice for the immediate purpose of suggesting that the more advanced state of collapse at a given blood pressure and blood volume loss in tourniquet shock as compared to hemorrhagic shock is probably attributable to the high blood viscosity in the former, i.e., that the critical state of the circulation in tourniquet shock is in part due to a qualitative change in the physical properties of the blood itself. Thus the therapy of tourniquet shock must differ from that of hemorrhagic shock in respect to the choice of a substance for blood volume replacement. The problem is one, as in burns, of restoring to normal as rapidly as possible, not only the blood volume, but also the proper proportion of blood constituents.

The effect of sodium succinate on 5-hour tourniquent shock in nembutalized dogs has been studied (17). Significant lowering of mortality was observed. Our data, showing the lack of a positive therapeutic effect of sodium succinate, are not strictly comparable to the above since we employed it in a more severe type of tourniquet shock in the absence of anesthesia. Nevertheless, since we found it possible to cure, by saline solution alone, 5 -hour tourniquet shock in which barbiturates were avoided, we regard the therapeutic benefit mentioned above as not related to the shock state per $s e$, but to the superimposed depressing effect of the barbiturates. Neutralization of barbiturate action by sodium succinate has been reported (18).

\section{CONCLUSIONS}

1. The application of tourniquets to both hind legs of unanesthetized dogs for 5 hours is not always followed by shock. If shock occurs, it is of moderate intensity. Saline solution given intravenously is curative.

2. The application of tourniquets to unanesthetized dogs for 8 to 11 hours will uniformly produce shock which is fatal if untreated. Intravenously administered plasma, 5 per cent bovine albumin in saline solution, or 25 per cent bovine albumin supplemented by peroral fluid are effective therapeutic agents, if the deficiency 
in plasma volume is made good while the blood pressure is above $60 \mathrm{~mm}$. $\mathrm{Hg}$. Occasionally, they may be effective at blood pressures between 60 and $40 \mathrm{~mm}$. Hg. Physiologic saline, 25 per cent albumin without peroral fluid, and 5 per cent saline with peroral water are not effective.

3. The critical blood pressure level of tourniquet shock is much higher than that of hemorrhagic shock. This may be related to the deleterious effect on cardiac output of the increased blood viscosity of tourniquet shock. Consequently, the high viscosity requires that plasma or plasma substitutes rather than whole blood be the agent of choice for blood volume replacement therapy.

4. Effective therapy is always accompanied by a substantial reduction in hematocrit and usually by a substantial restoration of the deficiency in plasma volume.

5. The course of events following ineffective though adequate blood volume replacement therapy of tourniquet shock is not materially altered by the administration of sodium succinate. Cure of 5-hour tourniquet shock, attributed by other investigators to succinic acid, is achieved by saline therapy alone in experiments in which anesthesia is omitted. It is therefore apparent that any value sodium succinate may have demonstrated in studies by other investigators may be attributable to its ability to counteract the depressing effects of barbiturates.

\section{BIBLIOGRAPHY}

1. Bailey, H., Surgery of Modern Warfare. Williams and Wilkins, Baltimore, 1941.

2. Wilson, H., and Roome, N. W., The effects of constriction and release of an extremity. An experimental study of the tourniquet. Arch. Surg., 1936, 32, 334.

3. Allen, F. M., Physical and toxic factors in shock. Arch. Surg., 1939, 38, 155.

4. Duncan, G. W., and Blalock, A., The effects of the application of a tourniquet on the general response to gross trauma to an extremity. Surgery, 1943, 13, 401.

5. Duncan, G. W., and Blalock, A., Shock produced by crush injury; effects of administration of plasma and local application of cold. Arch. Surg., 1942, 45, 183.

6. Warren, S., Personal communication.

7. Swingle, W. W., Remington, J. W., Kleinberg, W., Drill, V. A., and Eversole, W. J., An experimental study of the tourniquet as a method for inducing circulatory failure in the dog. Am. J. Physiol., 1942, 138, 156.

8. Seligman, A. M., Frank, H. A., and Fine, J., The relation of blood viscosity to cardiac output in traumatic shock. To be published.

9. Allen, F. M., Experiments on theory and therapy of shock. Arch. Phys. Therapy, 1943, 24, 327.

10. Warren, J. V., Merrill, A. J., and Stead, E. A., The rôle of the extracellular fluid in the maintenance of a normal plasma volume. J. Clin. Invest., 1943, 22, 635.

11. Shorr, E., Personal communication.

12. Fine, J., and Seligman, A. M., Traumatic shock. IV. A study of the problem of the "lost plasma" in hemorrhagic shock by the use of radioactive plasma protein. J. Clin. Invest., 1943, 22, 285.

13. Fine, J., and Seligman, A. M., Traumatic shock. An experimental study including evidence against the capillary leakage hypothesis. Ann. Surg., 1943, 118, 238.

14. Fine, J., and Seligman, A. M., Traumatic shock. VII. A study of the problem of the "lost plasma" in hemorrhagic, tourniquet, and burn shock by the use of radioactive iodo-plasma protein. J. Clin. Invest., 1944, 23, 720.

15. Goldberg, M., and Fine, J., Intestinal absorption in traumatic shock. To be published.

16. Whittaker, S. R. F., and Winton, F. R., The apparent viscosity of blood flowing in the isolated hind limb of the dog, and its variation with corpuscular concentration. J. Physiol., 1933, 78, 339.

17. Mylon, E., Winternitz, M. C., and deSüto-Nagy, G. J., Studies on therapy in traumatic shock. Am. J. Physiol., 1943, 139, 313.

18. Soskin, S., and Taubenhaus, M., Sodium succinate as an antidote for barbiturate poisoning and in the control of the duration of barbiturate anesthesia. J. Pharmacol. and Exper. Therap., 1943, 78, 49. 